\title{
PERFIL EPIDEMIOLÓGICO DOS PACIENTES INTERNADOS POR DOENÇA DE PARKINSON NO BRASIL NO ANO DE 2020
}

\author{
EPIDEMIOLOGICAL PROFILE OF PATIENTS HOSPITALIZED FOR \\ PARKINSON'S DISEASE IN BRAZIL IN 2020
}

Mateus Andrade Ferreira ${ }^{1}$

Caio Victor Queiroga Barreto ${ }^{2}$

Francisco Samuel Estrela Dantas ${ }^{3}$ Árnem Diógenes Bastos Bezerra ${ }^{4}$

Matheus da Silva Alves ${ }^{5}$

José Guilherme Ferreira Marques Galvão ${ }^{6}$

Resumo: Objetivo: Identificar o perfil epidemiológico dos pacientes internados por Doença de Parkinson no Brasil no ano de 2020. Metodologia: Trata-se de uma pesquisa documental e retrospectiva, com abordagem quantitativa, que utilizou como fonte de dados secundários as informações contidas no DataSUS, Informações de saúde - Epidemiológicas e Morbidade - Internações - Por local de internação. O período analisado foi o de janeiro de 2020 a dezembro de 2020. Para seleção dos casos, se utilizou o Lista de Morbidade CID-10: Doença de Parkinson, bem como a Projeção da População do Brasil e Unidades da Federação por sexo e idade do Instituto Brasileiro de Geografia e Estatística para os anos de 2010 a 2060 feita em 2018, a análise se deu por proporção, média e razão. Resultados: Os dados encontrados no sistema demonstraram uma prevalência dos casos em pessoas de raça branca, seguida pelas raças parda, negra, amarela e nenhum caso relatado na população indígena. Houve um aumento nos casos de Parkinson que acompanham o aumento da idade dos pacientes com seu pico de prevalência acima de 80 anos tanto para homens como para mulheres. Os números encontrados mostram um maior número de casos de doença de Parkinson na população

\footnotetext{
${ }^{1}$ Acadêmico de Medicina da Faculdade Santa Maria.

${ }^{2}$ Acadêmico de Medicina da Faculdade Santa Maria.

${ }^{3}$ Acadêmico de Medicina da Faculdade Santa Maria.

${ }^{4}$ Acadêmico de Medicina da Faculdade Santa Maria.

${ }^{5}$ Acadêmico de Medicina da Faculdade Santa Maria.

6 Farmacêutico generalista graduado pela Universidade Federal da Paraíba (UFPB). Mestre em ciências fisiológicas pelo Programa de Pós-graduação Multicêntrico em Ciências Fisiológicas (PPGMCF). Docente do curso de medicina da disciplina Imunologia da Faculdade Santa Maria (FSM).
} 
masculina (3,43 casos por 100.00 hab.) em comparação com a população feminina (2,69 casos por 100.00 hab.). Conclusão: Os resultados encontrados demonstraram que a Doença de Parkinson no Brasil afeta, em sua maioria, pessoas de raça branca. Soma-se a isso uma maior prevalência em pessoas de idade avançada que também possuem quadros mais graves da doença. Por fim, foram encontradas evidências que o público masculino está mais susceptível a desenvolver a doença, fato atribuído principalmente a ausência do hormônio estrogênio que possui ação protetora das células neuronais

Palavras Chave: Doença de Parkinson, Epidemiologia, Perfil de Saúde.

Abstract: Objective: To identify the epidemiological profile of patients hospitalized for Parkinson's Disease in Brazil in 2020. Methodology: This is a documentary and retrospective research, with a quantitative approach, which used the information contained in DataSUS as a secondary data source, - Health information Epidemiological and Morbidity - Admissions - By place of admission. The period analyzed was from January 2020 to December 2020. To select the cases, the ICD-10 Morbidity List: Parkinson's Disease was used, as well as the Projection of the Brazilian Population and Federation Units by sex and age of the Brazilian Institute of Geography and Statistics for the years 2010 to 2060 carried out in 2018, the137 analysis was carried out by proportion, average and ratio. Results: The data found in the system showed a prevalence of cases in white people, followed by brown, black, yellow and no cases reported in the indigenous population. There has been an increase in Parkinson's cases that accompany the increase in patient age, with its prevalence peak above 80 years for both men and women. The figures found show a greater number of cases of Parkinson's disease in the male population (3.43 cases per 100,000 inhab.) compared to the female population (2.69 cases per 100,000 inhab.). Conclusion: The results found showed that Parkinson's Disease in Brazil mostly affects white people. Added to this is a higher prevalence in elderly people who also have more severe cases of the disease. Finally, evidence shows that the male public is more likely to develop the disease, a fact mainly attributed to the absence of the estrogen hormone, which has a protective action on neuronal cells.

Keywords: Parkinson Disease, Epidemiology, Health Profile. 


\section{INTRODUÇÃO}

A Doença de Parkinson (DP) é a segunda doença neurodegenerativa mais comum no mundo, caracterizada por sintomas motores (tremores, bradicinesia, rigidez e instabilidade postural) que surgem devido ao acúmulo de corpos de Lewy contendo a-sinucleína na substância negra do cérebro (GARCIA-RUIZ; CHAUDHURI; MARTINEZ-MARTIN, 2014; GUO et al., 2013). Seu desenvolvimento também é marcado por sintomas não motores, dentre os quais se destacam a depressão, transtornos do sono, obstipação, comprometimento da memória e perda de peso (FERNANDES; ANDRADE FILHO, 2018).

Sua incidência aumenta com a idade da população - surge normalmente entre 50 e 80 anos com um pico aos 70 anos, atingindo $1-2 \%$ das pessoas com mais de 65 anos de idade e $4 \%$ da população com mais de 80 anos em todo o mundo. Dentro do grupo de pessoas com Parkinson, aproximadamente 6 em cada 100 desenvolveram a doença antes dos 50 anos (TANG et al., 2016). Além disso, ela é mais comumente encontrada em homens e sofre influência de diversos fatores físicos e ambientais como genes, hormônios e países de residência (CABREIRA; MASSANO, 2019).

Atualmente, não existem tratamentos capazes de cessar os efeitos neurodegenerativos ou restaurar os neurônios perdidos no curso da doença. Isso faz com que a DP se torne uma doença crônica grave que exige um tratamento clínico individualizado feito por uma equipe multiprofissional com objetivo de diminuir os impactos do quadro sobre os indivíduos (CABREIRA; MASSANO, 2019).

Dessa forma é importante que se estabeleça o perfil clínico e epidemiológico dos indivíduos acometidos com a DP em cada país, de modo que possam ser identificadas as principais características que a doença assume em cada população para que ela possa ser diagnosticada precocemente e tratada de maneira mais eficaz. 


\section{OBJETIVO}

Identificar o perfil epidemiológico dos pacientes internados por Doença de Parkinson no Brasil no ano de 2020.

\section{MÉTODO}

Trata-se de uma pesquisa documental e retrospectiva, com abordagem quantitativa, que utilizou como fonte de dados secundários as informações contidas no DataSUS, - Informações de saúde - Epidemiológicas e Morbidade - Internações Por local de internação. O período analisado foi o de janeiro de 2020 a dezembro de 2020. Para seleção dos casos, se utilizou o Lista de Morbidade CID-10: Doença de Parkinson, bem como a Projeção da População do Brasil e Unidades da Federação por sexo e idade do Instituto Brasileiro de Geografia e Estatística para os anos de 2010 a 2060 feita em 2018.

As variáveis de análise foram casos notificados por idade (agrupadas nas faixas etárias de 0 a 49 anos, 50 a 59 anos, 60-69 anos, 70-79 anos e acima de 80 anos), sexo (masculino e feminino) e raça (branca, preta, parda, amarela, indígena).

As informações colhidas foram inseridas no software Microsoft Excel 2017, onde foram analisadas e representadas graficamente para apresentação dos dados. $A$ análise se deu por proporção, média e razão. $O$ uso das informações da base de dados do DataSUS é livre a toda população brasileira e certificam os princípios éticos contidos na resolução $N^{\circ}$ 466/2012 do Conselho Nacional de Saúde (CNS), não havendo, portanto, a necessidade de submissão a um Comitê de Ética. Não obstante, foram adotados os princípios éticos de se preservar a fidedignidade dos dados utilizados. 


\section{RESULTADOS E DISCUSSÃO}

\section{RAÇA}

\section{Internações por DP segundo Cor/raça 2020}

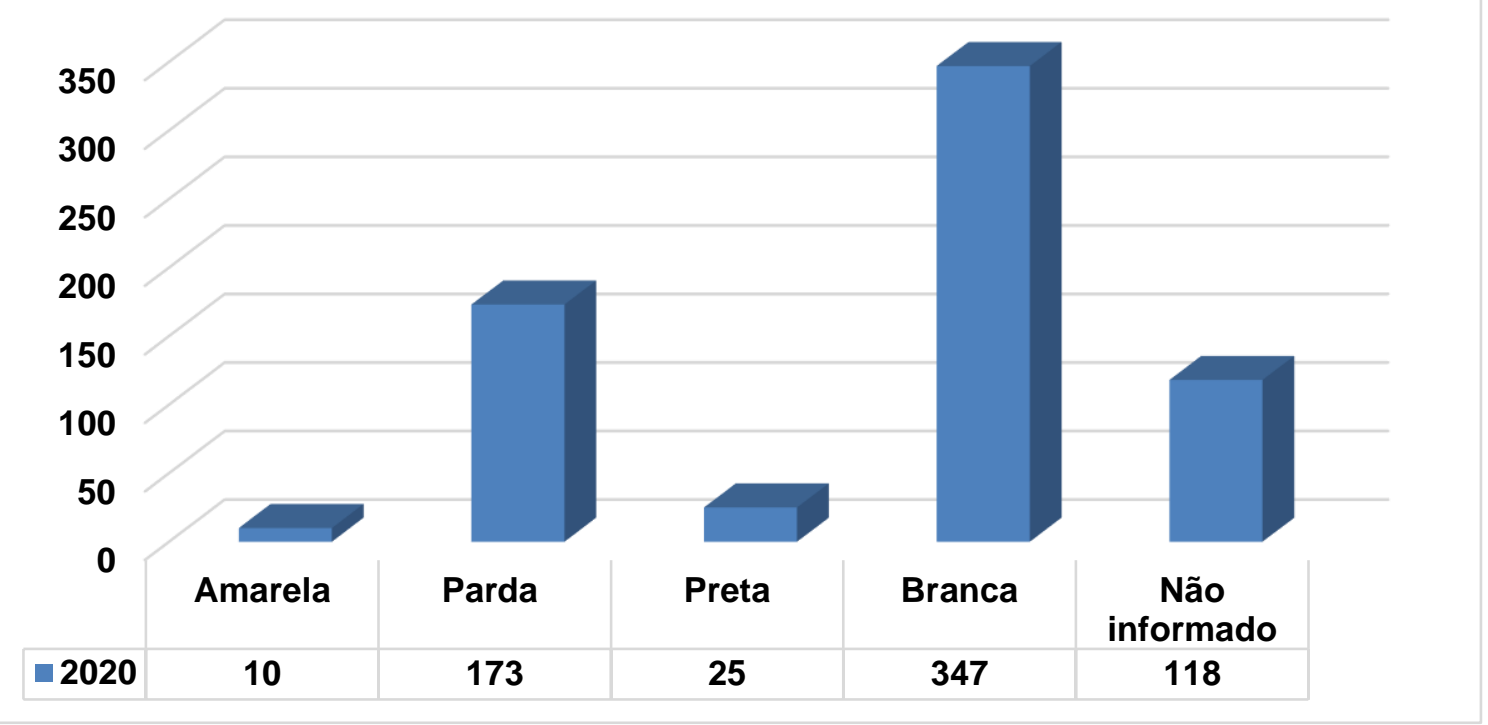

Fonte: Elaboração própria. Dados: Ministério da Saúde - Sistema de Informações Hospitalares do SUS (SIH/SUS).

Os dados encontrados no sistema demonstraram uma prevalência dos casos em pessoas de raça branca, seguida pelas raças parda, negra, amarela e nenhum caso relatado na população indígena. Esses dados corroboram a pesquisa feita por Melo et. al (2018) entre os anos de 2015 e 2016 que mensurou a mesma prevalência entre as raças.

O mesmo estudo deu destaque ao grande número de casos subnotificados que não tiveram esse quesito preenchido em sua ficha, chegando a quase $20 \%$ dos casos. Esse fenômeno compromete o planejamento das ações de intervenção, uma vez que a análise dos dados se torna menos fidedigna à realidade.

Estudos relacionando raça ao desenvolvimento da DP ainda se mostram escassos, salvo a pesquisa realizada por Santos e Milagras (2015), que alia essa distribuição à maior expectativa de vida alcançada por pessoas de raça branca (a 
idade é um fator comprovado para maior desenvolvimento da DP). Entretanto, os dados encontrados através do Sistema de Informações Hospitalares do SUS (SIH/SUS) mostram que, mesmo na população mais jovem (menos de 60 anos), a diferença entre raças ainda é proeminente: Branca (97); Preta (7); Parda (62); Amarela (3); sem informação (26).

IDADE

\section{Internações por DP segundo Faixa Etária e sexo 2020}

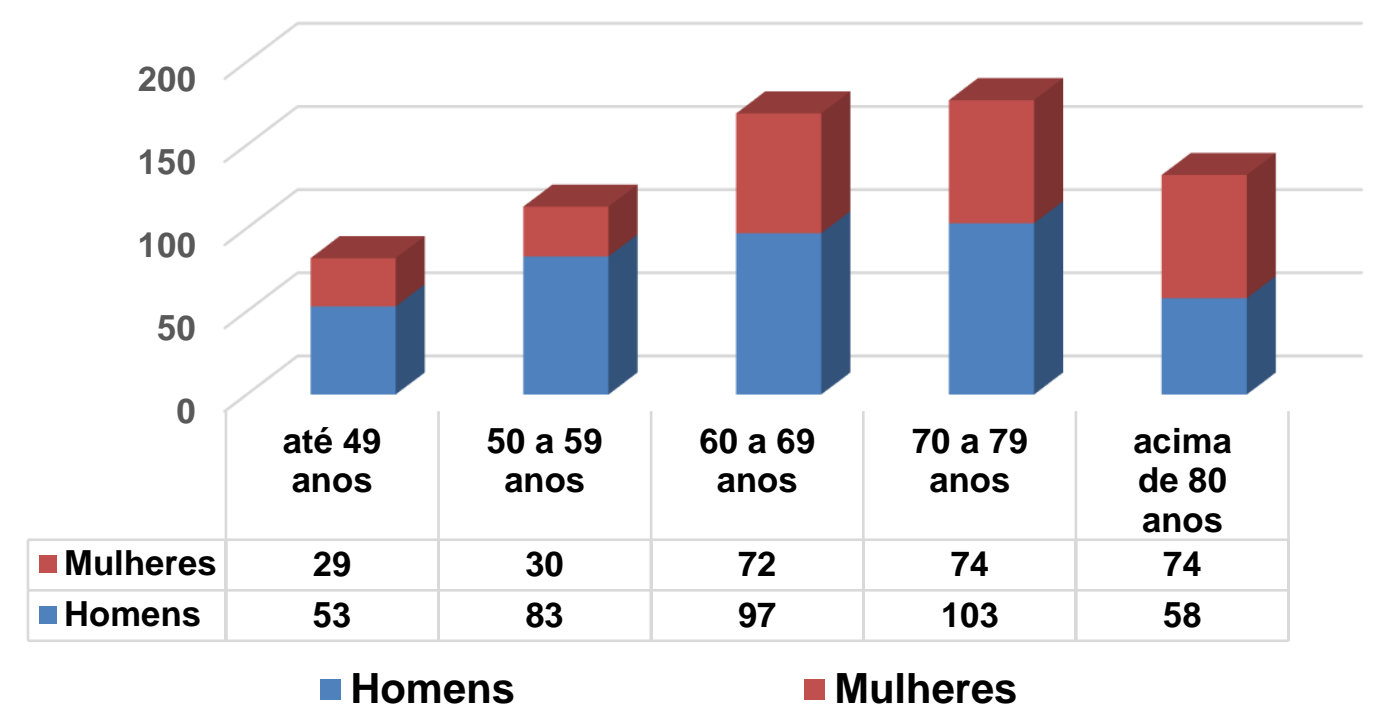

Fonte: Elaboração própria. Dados: Ministério da Saúde - Sistema de Informações Hospitalares do SUS (SIH/SUS).

Para descobrir a taxa de prevalência foi utilizada a Projeção da população do Brasil e Unidades da Federação por sexo e idade do Instituto Brasileiro de Geografia e Estatística (2018) junto aos dados obtidos no DATASUS encontrados na tabela 1. 
Tabela 1: Incidência de Parkinson na População Brasileira 2020.

\begin{tabular}{|c|c|c|c|}
\hline Idade & População & $\begin{array}{c}\text { Internações por } \\
\text { Doença de Parkinson }\end{array}$ & $\begin{array}{c}\text { Prevalência de Doença } \\
\text { de Parkinson }\end{array}$ \\
\hline Até 49 anos & $\begin{array}{l}29.255 .478 \\
\text { hab. }\end{array}$ & 82 internações & $\begin{array}{c}0,28 \text { casos por } 100.00 \\
\text { hab. }\end{array}$ \\
\hline 50 a 59 anos & $\begin{array}{l}23.875 .081 \\
\text { hab. }\end{array}$ & 113 internações & $\begin{array}{c}0,47 \text { casos por } 100.00 \\
\text { hab. }\end{array}$ \\
\hline 60 a 69 anos & $\begin{array}{l}16.732 .972 \\
\text { hab. }\end{array}$ & 169 internações & 1 casos por 100.00 hab. \\
\hline 70 a 79 anos & $\begin{array}{l}9.023 .052 \\
\text { hab. }\end{array}$ & 177 internações & $\begin{array}{c}1,96 \text { casos por } 100.00 \\
\text { hab. }\end{array}$ \\
\hline $\begin{array}{l}\text { Acima de } 80 \\
\text { anos }\end{array}$ & $\begin{array}{l}4.441 .053 \\
\text { hab. }\end{array}$ & 132 internações & $\begin{array}{c}2,97 \text { casos por } 100.00 \\
\text { hab. }\end{array}$ \\
\hline
\end{tabular}

Fonte: IBGE - Projeção da população do Brasil e Unidades da Federação por sexo e idade para o período 2010-2060. Ministério da Saúde - Sistema de Informações Hospitalares do SUS (SIH/SUS).

Os dados encontrados demonstram um aumento nos casos de Parkinson que acompanham o aumento da idade dos pacientes com seu pico de prevalência acima de 80 anos tanto para homens como para mulheres (tabela 2). Em contrapartida, a revisão sistemática realizada por Hirsch et al. (2016), que avaliou estudos epidemiológicos de DP entre 2001 e 2014, localiza o pico de casos entre mulheres na idade de 70 a 79 anos; já para os homens, o pico coincidiu com os dados do DATASUS, sendo encontrada no grupo de pessoas maiores de 80 anos. Ademais, 0 estudo feito por Santos e Milagras (2015) relatou um aumento dos casos a partir de 60 anos encontrando declínio a partir de 80 anos.

A explicação para esse fenômeno encontra-se na relação entre a idade do indivíduo e a piora dos sintomas que ele apresenta conforme envelhece, devido a alterações nos genes reguladores do ciclo celular nos neurônios da parte compacta da substância negra, que são associadas ao envelhecimento dos astrócitos e à perda funcional e estrutural dos tecidos dessa região nos pacientes com DP (KRITSILIS et al, 2018).

A relação entre idade e Parkinson é ainda mais importante do que a duração absoluta da doença, traduzida no fato de que idade mais avançada de início está 
relacionada a disfunções cognitivas mais graves do que um maior tempo de doença nos casos que ela começa de maneira precoce (TANG et al., 2016).

Mesmo que a Doença de Parkinson seja mais comum e grave em pacientes mais velhos, é importante destacar os casos em faixas etárias menores (menores que 50 anos) que chegam a valores consideráveis como mostrado no Gráfico 1 com $12,1 \%$ dos casos. Isso corrobora o estudo de Arshad et al. (2017) que encontrou $11,3 \%$ dos pacientes com Parkinson na faixa etária até 50 anos.

Apesar da relação de gravidade dos sintomas e idade, os casos de doenças em indivíduos jovens são bastante preocupantes. Esses pacientes passam a sofrer dos sintomas comuns à doença, como discinesia e distonia, desde cedo, cooptando uma parte considerável de suas vidas (FERGUSON; RAJPUT; RAJPUT, 2015).

Cabreira e Massano (2019) argumentam em seu estudo que essa maior prevalência da doença encontrada em idades elevadas se deve também ao melhor reconhecimento da doença, aumentando o número de diagnósticos, e a um tratamento mais eficaz, que garantiu uma maior sobrevivência após 60 anos, saindo de uma média de 9,4 anos de sobrevida antes da levodopa e chegando a uma média de 14,6 anos atualmente, mais próxima da média da população em geral que vive 23,3 anos.

SEXO

Tabela 2: Incidência de Parkinson na População Brasileira 2020 por sexo.

\begin{tabular}{cccc}
\hline Idade & População & $\begin{array}{c}\text { Internações } \\
\text { por Doença } \\
\text { de Parkinson }\end{array}$ & $\begin{array}{c}\text { Prevalência de Doença de } \\
\text { Parkinson }\end{array}$ \\
\hline $\begin{array}{c}\text { Até } \mathbf{4 9} \\
\text { anos } \\
\text { (Homens) }\end{array}$ & 14.151 .255 hab. & 53 internações & 0,37 casos por 100.00 hab. \\
\hline $\begin{array}{c}\mathbf{5 0} \text { a } 59 \\
\text { anos }\end{array}$ & 11.347 .109 hab. 83 internações & 0,73 casos por 100.00 hab. \\
$\begin{array}{c}\text { (Homens) } \\
\begin{array}{c}\mathbf{6 0} \text { a } \mathbf{6 9} \\
\text { anos }\end{array}\end{array}$ & 7.687 .563 hab. & 97 internações & 1,26 casos por 100.00 hab. \\
(Homens) & 7.940 .883 hab. & 103 & 2,61 casos por 100.00 hab. \\
\hline $\mathbf{7 0 ~ a ~} 79$ & 3
\end{tabular}




\begin{tabular}{|c|c|c|c|}
\hline $\begin{array}{c}\text { anos } \\
\text { (Homens) }\end{array}$ & & internações & \\
\hline $\begin{array}{c}\text { Acima de } \\
80 \text { anos } \\
\text { (Homens) }\end{array}$ & 1.689.049 hab. & 58 internações & 3,43 casos por 100.00 hab. \\
\hline $\begin{array}{c}\text { Até } 49 \\
\text { anos } \\
\text { (Mulheres) }\end{array}$ & 15.104.223 hab. & 29 internações & 0,19 casos por 100.00 hab. \\
\hline $\begin{array}{c}50 \text { a } 59 \\
\text { anos } \\
\text { (Mulheres) }\end{array}$ & 12.527.972 hab. & 30 internações & 0,24 casos por 100.00 hab. \\
\hline $\begin{array}{c}60 \text { a } 69 \\
\text { anos } \\
\text { (Mulheres) }\end{array}$ & 9.045.409 hab. & 72 internações & 0,80 casos por 100.00 hab. \\
\hline $\begin{array}{c}70 \text { a } 79 \\
\text { anos } \\
\text { (Mulheres) }\end{array}$ & 5.082.169 hab. & 74 internações & 1,45 casos por 100.00 hab. \\
\hline $\begin{array}{c}\text { Acima de } \\
80 \text { anos } \\
\text { (Mulheres) }\end{array}$ & 2.752.004 hab. & 74 internações & 2,69 casos por 100.00 hab. \\
\hline
\end{tabular}

Fonte: IBGE - Projeção da população do Brasil e Unidades da Federação por sexo e idade para o período 2010-2060. Ministério da Saúde - Sistema de Informações Hospitalares do SUS (SIH/SUS).

Os dados encontrados mostram um maior número de casos de doença de Parkinson na população masculina (3,43 casos por 100.00 hab.) em comparação com a população feminina (2,69 casos por 100.00 hab.) situação confirmada por Campos et al., (2015) no seu estudo realizado com pacientes do Ambulatório de Distúrbios do Movimento da Universidade Estadual de Campinas. Fernandes e Andrade Filho (2018) e Podcasy e Epperson (2016) atribuem essa discrepância ao hormônio sexual feminino (estrógeno) que seria um fator protetor para as células neuronais, tornando a doença mais leve, enquanto Hirsch et al. (2016) expande essa teoria ao afirmar que a ausência desse hormônio nos homens leva a uma maior taxa de mortalidade.

As diferenças entre os sexos se estendem também para os sintomas motores e não motores. Segundo Guo et al. (2013), homens apresentam mais sintomas urinários e dificuldades em ter relações sexuais enquanto as pacientes femininas encontraram uma maior dificuldade pra dormir, sintomas de humor, apatia e uma 
maior sensação de dor. Quanto aos sintomas motores, os homens apresentam maior rigidez motora e tremores maiores, enquanto mulheres tendem a ter mais tremores leves e discinesia.

Tysnes e Storstein (2017) mostram que o risco aumentado de adquirir DP em homens pode estar relacionado com um aumento no uso de cigarros no fim do século XX, além de uma maior poluição do ar relacionada ao tráfego. Já Santos e Milagras (2015) atribuem essa maior prevalência ao fator do estresse físico e emocional que os homens enfrentam durante a vida, além do já citado efeito protetor do estrogênio em mulheres.

\section{CONCLUSÃO}

Os dados demonstraram que a Doença de Parkinson no Brasil afeta, em sua maioria, pessoas de raça branca. Soma-se a isso uma maior prevalência em pessoas de idade avançada que também possuem quadros mais graves da doença. Por fim, foram encontradas evidências que o público masculino está mais susceptível a desenvolver a doença, fato atribuído principalmente a ausência do hormônio estrogênio que possui ação protetora das células neuronais.

Vale ressaltar que esse estudo utilizou apenas dados hospitalares o que somado aos casos de subnotificação e aos estudos que utilizam diferentes fontes de pacientes (em todos os níveis de atenção à saúde) limita as conclusões quanto ao perfil dos pacientes com Doença de Parkinson. 


\section{REFERÊNCIAS BIBLIOGRÁFICAS}

1. ARSHAD, $Q$ et al. Biased numerical cognition impairs economic decision-making in Parkinson's disease. Ann Clin Transl Neurol, vol. 4, p.739-748. 2017. https://doi.org/10.1002/acn3.449. Disponível em: https://onlinelibrary.wiley.com/doi/full/10.1002/acn3.449. Acesso em: 02/12/2020.

2. BOVOLENTA, Tânia Maria; FELICIO, André Carvalho. Parkinson's patients in the Brazilian Public Health Policy context. Einstein (São Paulo), São Paulo, v. 14, n. 3, p. 7-9, Sept. 2016. Availablefrom $<$ http://www.scielo.br/scielo.php?script=sci_arttext\&pid=S167945082016000300001\&lng=en\&nrm=iso>. access on 02 Dec. 2020. https://doi.org/10.1590/S1679-45082016ED3780.

3. CAMPOS, Lidiane S. et al. Clinical predictors of cognitive impairment and psychiatric complications in Parkinson's disease. Arq. Neuro-Psiquiatr., São Paulo, v. 73, n. 5, p. 390-395, May 2015. Available from <http://www.scielo.br/scielo.php?script=sci_arttext\&pid=S0004$282 X 2015000500004 \&$ Ing=en\&nrm=iso $>$. access on 04 June 2020. https://doi.org/10.1590/0004$282 \times 20150016$.

4. FERGUSON LW, RAJPUT AH, RAJPUT A. Early-onset vs. Late-onset Parkinson's disease: A Clinical-pathological Study. Can J Neurol Sci. Vol. 43, n. 1, p. 113-9. 2016. doi: 10.1017/cjn.2015.244. Disponível em: https://pubmed.ncbi.nlm.nih.gov/26189779/. Acesso em: 02/12/2020.

5. FERNANDES, Itana; ANDRADE FILHO, Antônio de Souza. Estudo Clínico-Epidemiológico de Pacientes com Doença de Parkinson em Salvador-Bahia. Revista Brasileira de Neurologia e Psiquiatria. Vol. 22, n. 1, p:45-59. 2018. Disponível em: https://www.revneuropsiq.com.br/rbnp/article/view/244/141. Acesso em: 02/12/2020.

6. GARCIA-RUIZ PJ, CHAUDHURI KR, MARTINEZ-MARTIN P. Non-motor symptoms of Parkinson's disease A review. J Neurol Sci. vol. 338, n. 1-2. p. 30-3. 2014. doi: 10.1016/j.jns.2014.01.002.

7. HIRSCH L et al. The Incidence of Parkinson's Disease: A Systematic Review and MetaAnalysis. Neuroepidemiology. Vol. 46, n. 4, p. 292-300. 2016. doi: 10.1159/000445751. Disponível em: https://www.karger.com/Article/FullText/445751. Acesso em: 02/12/2020.

8. INSTITUTO BRASILEIRO DE GEOGRAFIA E ESTATÍSTICA (IBGE). Diretoria de Pesquisas. Coordenação de População e Indicadores Sociais. Gerência de Estudos e Análises da Dinâmica Demográfica. Projeção da população do Brasil e Unidades da Federação por sexo e idade para o período 2010-2060. Disponível em: https://www.ibge.gov.br/estatisticas/sociais/populacao/9109-projecao-dapopulacao.html?=\&t=resultados. Acesso em: 09/02/2020.

9. KRITSILIS M, V et al. Aging, Cellular Senescence and Neurodegenerative Disease. Int $\mathbf{J}$ Mol Sci. Vol. 19. N. 10. P. 2937. 2018. doi: 10.3390 / ijms19102937. Disponível em: https://pubmed.ncbi.nlm.nih.gov/30261683/. Acesso em: 02/12/2020.

10. MELO, Maria Aparecida de Souza et. al. Percepção dos profissionais de saúde sobre os fatores associados à subnotificação no Sistema Nacional de Agravos de Notificação. Revista de Administração em Saúde. São Paulo. Vol. 18. № 71. Pág 1-17. 2018. http://dx.doi.org/10.23973/ras.71.104. Acesso em 19 de junho de 2020.

11. PODCASY JL, EPPERSON CN. Considering sex and gender in Alzheimer disease and 
other dementias. Dialogues Clin Neurosci. Vol. 18, n. 4, p. 437-446. 2016.

12. TYSNES, Ole-Bjørn; STORSTEIN, Anette. Epidemiology of Parkinson's disease. Journal of Neural Transmission, v. 124, n. 8, p. 901-905, 2017. https://link.springer.com/article/10.1007/s00702-017-1686-y.

13. CABREIRA, Verónica; MASSANO, João. Doença de Parkinson: Revisão Clínica e Atualização. Acta Med Port. Vol. 32, n. 10, p. 661-670, 2019. Disponível em: https://actamedicaportuguesa.com/revista/index.php/amp/article/view/11978/5774. Acesso em: 03/12/2020.

14. SANTOS, Viviani Lara; MILAGRAS, Bruno Silva. Perfil Epidemiológico da Doença de Parkinson no Brasil. Trabalho de Conclusão de Curso (Curso de Bacharelado em Biomedicina) - Faculdade de Ciências da Educação e Saúde Graduação em Biomedicina, Centro Universitário de Brasília. BRASíLIA, p.21, 2015.2 Disponível em: https://repositorio.uniceub.br/jspui/handle/235/6857. Acesso em: 03/12/2020.

15. TANG, $\mathrm{H}$ et al. Cognitive profile of Parkinson's disease patients: a comparative study between early-onset and late-onset Parkinson's disease. Int J Neurosci. Vol. 126 n. 3 p. $227-$ $234.2016 . \quad$ doi: 10.3109/00207454.2015.1010646. Disponível: https://pubmed.ncbi.nlm.nih.gov/26001202/. Acesso em: 04/12/2020.

16. GUO, $\mathrm{X}$ et al. Gender and onset age-related features of non-motor symptoms of patients with Parkinson's disease - a study from Southwest China. Parkinsonism Relat Disord. Vol. 19, n. 11, p. 961-965. 2013. doi:10.1016/j.parkreldis.2013.06.009. Disponível em: https://pubmed.ncbi.nlm.nih.gov/23849500/. Acesso em: 04/12/2020. 